\title{
Cardiac Paraganglioma
}

National Cancer Institute

\section{Source}

National Cancer Institute. Cardiac Paraganglioma. NCI Thesaurus. Code C6410.

A benign or malignant extra-adrenal parag ang lioma arising from paragang lia located in the heart. 